\title{
A truncated form of the Carbon catabolite repressor 1 increases cellulase production in Trichoderma reesei
}

\author{
Thiago M Mello-de-Sousa ${ }^{1 \dagger}$, Rita Gorsche ${ }^{1 \dagger}$, Alice Rassinger ${ }^{1}$, Marcio J Poças-Fonseca ${ }^{2}$, Robert L Mach ${ }^{1}$
} and Astrid R Mach-Aigner ${ }^{1 *}$

\begin{abstract}
Background: Rut-C30 is a cellulase-hyperproducing Trichoderma reesei strain and, consequently, became the ancestor of most industry strains used in the production of plant cell wall-degrading enzymes, in particular cellulases. Due to three rounds of undirected mutagenesis its genetic background differs from the wild-type QM6a in many ways, of which two are the lack of a $83 \mathrm{~kb}$ large sequence in scaffold 15 and the partial lack of the gene encoding the Carbon catabolite repressor 1 (CREI). However, it is still unclear, what exactly enhances cellulase production in Rut-C30.

Results: The investigation of the expression of two genes encoding cellulases (cbh1 and cbh2) and the gene encoding their main transactivator ( $x y r 1$ ) revealed that the presence of the truncated form of CREI (CREI-96) contributes more to the Rut-C30 phenotype than a general loss of CREI-mediated carbon catabolite repression (crel deletion strain) or the deletion of 29 genes encoded in the scaffold 15 (83 kb deletion strain). We found that the remaining cre 1 in Rut-C30 (cre1-96) is transcribed into mRNA, that its putative gene product (Cre1-96) is still able to bind DNA, and that the CREI-binding sites in the upstream regulatory regions of the chosen CREl-target genes are still protected in Rut-C30. As it was previously reported that CREI acts on the nucleosome positioning, we also analyzed chromatin accessibility of the core promoters of CREl-target genes and found them open even on D-glucose in the presence of CREI-96.

Conclusions: The lack of the full version of CREI in Rut-C30 corresponds with a partial release from carbon catabolite repression but is not completely explained by the lack of CREI. In contrast, the truncated CREI-96 of Rut-C30 exerts a positive regulatory influence on the expression of target genes. Mechanistically this might be explained at least partially by a CREl-96-mediated opening of chromatin.
\end{abstract}

Keywords: Trichoderma reesei, Hypocrea jecorina, Rut-C30, Cellulases, Carbon catabolite repressor 1, Chromatin

\section{Background}

The filamentous ascomycete Trichoderma reesei is a saprophyte known for its ability to efficiently degrade biomass material by plant cell wall (PCW)-degrading enzymes. A genome-wide analysis identified 10 cellulolytic and 16 hemicellulolytic enzyme-encoding genes in $T$. reesei [1], of which the two most prominent cellulosedegrading enzymes are the cellobiohydrolases $\mathrm{CBHI}$ and CBHII (EC 3.2.1.91) [2]. However, the defining feature of

\footnotetext{
* Correspondence: astrid.mach-aigner@tuwien.ac.at

${ }^{\dagger}$ Equal contributors

'Department for Biotechnology and Microbiology, Institute of Chemical Engineering, Vienna University of Technology, Gumpendorfer Str. 1a, A-1060 Wien, Austria

Full list of author information is available at the end of the article
}

this fungus is the exceedingly high amount of secreted enzymes that provoked its industrial exploitation for their production. Next to the pulp and paper industry [3], the food and feed industry [4], and the textile industry [5], these enzymes are applied in the production of biofuels [4]. With regards to cellulosic ethanol, the production costs of the needed enzymes massively influence the price and the competitiveness of the end-product. As a result their efficient expression remains an important topic of research.

The ancestor of most current industry strains is Rut-C30 [6,7]. It was derived from the $T$. reesei wild-type isolate QM6a through three rounds of mutagenesis (ultraviolet (UV) light and N-nitroguanidine) followed by a screening 
for the release from carbon catabolite repression (CCR) and high cellulase activity [8-10]. Since then the study of the specific physiological and genetic changes in Rut-C30 has been of interest $[7,11]$ and a number of properties have been identified, for example, a $83 \mathrm{~kb}$ large region located in scaffold 15, which encodes 29 genes is lacking in Rut-C30 $[12,13]$. Another important property of Rut-C30 is the lack of the full version of the Carbon catabolite repressor 1 (CREI, [14]) having left a cre1 sequence that would only encode for one of the two zinc finger regions of CREI (96 aa long) [15]. The native CREI is a $\mathrm{C}_{2} \mathrm{H}_{2}$-type zinc finger protein with the consensus sequence 5'-SYGGRG-3' [14], and is orthologous to CreA from Aspergillus sp. [16-18] and MIG1 from Saccharomyces cerevisiae [19].

In T. reesei CREI is known to act on a number of regulatory levels. Firstly, it directly represses transcription of several genes encoding for PCW-degrading enzymes, for example the cbh1 gene [20], by binding to tandem and inverted repeats in their upstream regulatory regions (URRs) $[20,21]$. Secondly, in the presence of D-glucose it represses the expression of the main transactivator of PCW-degrading enzyme expression, the Xylanase regulator 1 (XYRI) [22,23]. XYRI is a Gal4-like Zn2Cys6 binuclear cluster protein, of which the expression can be induced by sophorose [6] or as above-mentioned, repressed by D-glucose. Notably, the extent of induction of xyr1 gene expression directly correlates with the induction of $c b h 1$ and $c b h 2$ gene expression, which is not the case for other genes in the XYRI regulon [6]. As a third regulatory level, CREI has been reported to play an essential role in correct nucleosome positioning, for example in the promoters of the cbh1 and $c b h 2$ genes [24,25]. Altogether, CREI plays a major role in the regulation of 250 genes, both in a repressing and inducing way [26].

In this study we investigated to which extent the two mentioned striking genetic properties of Rut-C30, namely the lack of the $83 \mathrm{~kb}$ in scaffold 15 and the absence of a full CREI contribute to its cellulase hyper-producing phenotype. A special focus was studying the role of the remaining truncated version of CREI with regard to the transcriptional regulation of target gene expression, the ability of DNA binding, and the influence on the chromatin structure. The PCW-degrading enzyme-encoding genes $c b h 1$ and $c b h 2$, as well as $x y r 1$ as the gene encoding their transactivator, were chosen as the CREI-target genes to be analyzed.

\section{Results}

The expression profile of $c b h 1, c b h 2$, and $x y r 1$ in QM6a-CREl ${ }_{96}$ is closest to Rut-C30

In order to find out which genetic properties of Rut-C30 contribute to its cellulase hyper-producing phenotype we compared its expression profile to three transgenic strains. Two of them bear Rut-C30-related mutations, namely $\Delta$ scaff 15 , which is a QM6a-derived strain with an $83 \mathrm{~kb}$ deletion in scaffold 15, and QM6a-CREI ${ }_{96}$, which bears the truncated CREI version like Rut-C30 does. We also included a QM6a-derived cre1 deletion strain because the phenotype of Rut-C30 is sometimes associated with the lack of CREI. We investigated expression levels of $c b h 1, c b h 2$, and xyr 1 under sophoroseinducing conditions. Interestingly, the transcript levels of all three genes were most similar in QM6a-CREI $I_{96}$ compared to Rut-C30, while in the case of the other two strains ( $\Delta$ cre 1 and $\Delta$ scaff 15$)$ considerably lower levels were detected (Figure 1a, b, c). This result raised the consideration that regulatory molecular mechanisms are related to the truncated version of cre 1 remaining in the Rut-C30 genome.

CREI sites in upstream regulatory regions of target genes are strongly protected from DNA methylation in Rut-C30

All above-mentioned analyzed genes (cbh1, cbh2, and xyr1) are under the regulatory influence of CREI [20,22,25]. To learn if the protection patterns against DNA methylation of the URRs bearing CREI sites of these genes differ between the wild-type QM6a and Rut-C30, we performed in vivo/ in vitro footprinting analyses. URRs of all three genes were investigated after both strains were pre-grown on glycerol and then incubated on D-glucose for 3 hours followed by dimethyl sulphate (DMS)-induced in vivo methylation. The footprinting pattern obtained for all genes showed the same or even stronger DNA occupancy in Rut-C30 compared to QM6a (Figure 2a, b, c). This finding prompted us to analyze if the truncated cre1 (cre1-96) in Rut-C30 is transcribed. Thus, after pre-growth both strains were transferred to media containing D-glucose as a repressing condition, sophorose as an inducing condition, and no carbon source as a control condition respectively, and incubated for 3 hours. While we detected a low basal transcript level (originating from the native cre1 gene) in the wild-type, we found increased levels in Rut-C30, whereupon the increase was more pronounced on D-glucose and sophorose than without carbon source (Figure 3a). Since the cre1-96 mRNA could be detected in high amounts in Rut-C30 regardless of the applied condition (D-glucose or sophorose), we again performed in vivo/in vitro footprinting to investigate if the DNA protection pattern changes condition-dependent. We analyzed the same URRs of $c b h 1, c b h 2$, and $x y r 1$ genes comparing the application of D-glucose and sophorose. As can be inferred from Figure $3 b$, c, d no conditionspecific differences could be detected even though strong DNA occupancy at the CREI sites was observed. This fits the expression results and suggests that CREI96 may no longer act in a D-glucose specific manner. 


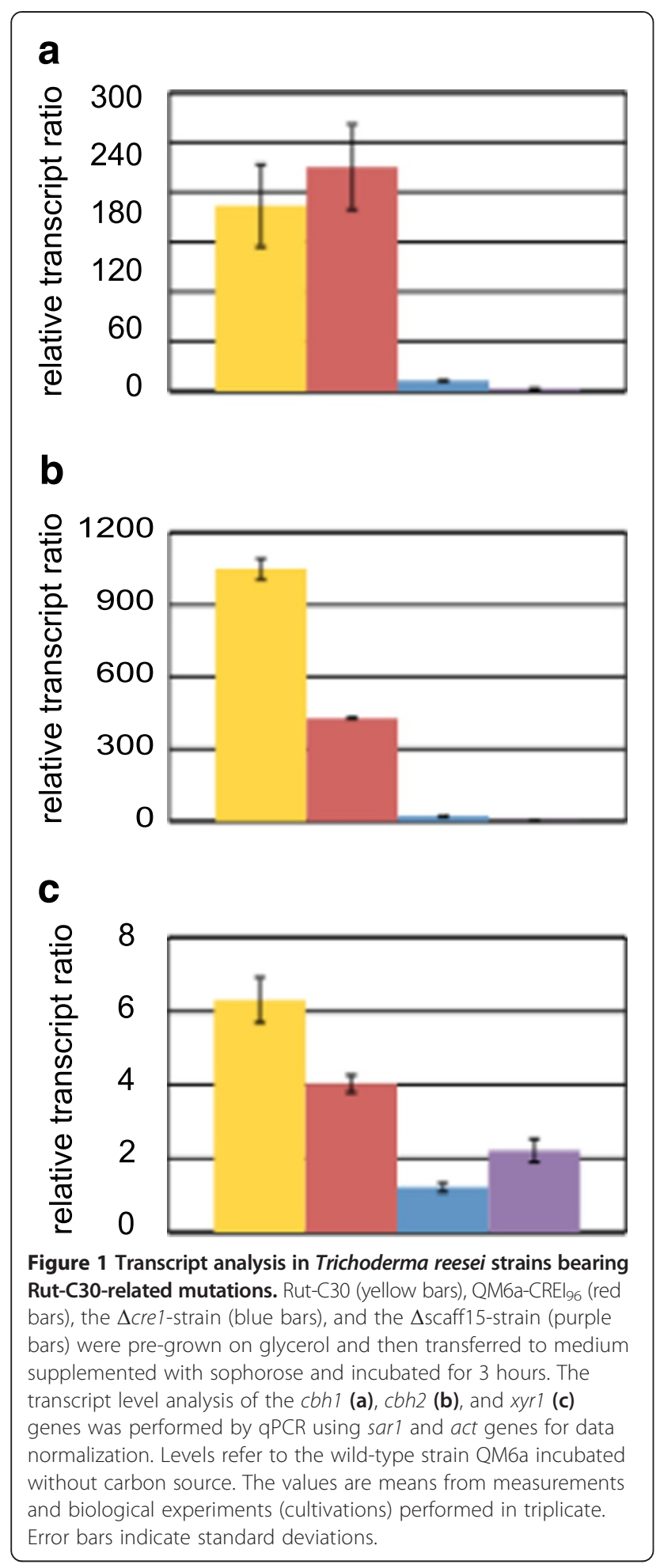

\section{The truncated CREI-96 protein of Rut-C30 can bind to DNA in vitro}

The results described above raise the question of whether the putative CREI-96 protein is still able to bind to DNA, especially because it lacks one of the two zinc fingers. To answer this question, we performed an electrophoretic mobility shift assay (EMSA) using the URR of cbh1 covering three functional CREI-binding sites as a probe (Figure 4a). The applied CREI-96 protein was heterologously expressed as GST-fusion protein and thrombincleaved before usage. The assay yielded two bands (Figure $4 \mathrm{~b}$ ), which represent most likely the binding of one or more CREI-96 proteins to the sites of the probe. Similar results were observed before reporting that shorter versions of CREI can still bind target DNA sequences yielding more than one band [28]. However, both complexes are specifically formed because the bands were diminished by adding a cold competitor, while they remained unchanged by adding a specifically mutated competitor (Figure 4b). Altogether, this supports the working hypothesis that the truncated CREI-96, which is putatively formed in Rut-C30, is still able to bind its DNA target sequences. It should be noted that in filamentous fungi other regulatory proteins are known that are also able to bind their DNA with a single zinc finger, such as the Aspergillus nidulans AreA [29].

\section{Higher gene expression corresponds with more open chromatin in QM6a-CREI 96}

As we had strong indication that the truncated version of CREI-96 is present in Rut-C30 and still is able to bind the URRs of its target genes, we aimed to get more insights on its potential regulatory role. Consequently, QM6a-CREI $I_{96}$ and the cre1 deletion strain were grown on D-glucose and samples were drawn after $36,39,42$, and 45 hours of cultivation. Because CREI was reported to be involved in nucleosome positioning within the cbh1 and the cbh2 promoter of $T$. reesei $[24,25]$, we investigated the chromatin packaging by applying chromatin accessibility real-time PCR (CHART-PCR) analysis of the core promoter regions of the $c b h 1, c b h 2$, and $x y r 1$ genes. Second, we investigated the expression of these genes by RT-qPCR to see if there was a correlation of transcript levels with the chromatin accessibility. We could detect a higher expression of $c b h 1$ and $c b h 2$ genes in QM6a-CREI ${ }_{96}$ compared to the $\Delta$ cre1-strain, which corresponded with a more open chromatin in the core promoters of the two genes in QM6a-CREI 96 (Figure $5 \mathrm{a}, \mathrm{b})$. The higher transcript levels of cellulaseencoding genes in QM6a-CREI 96 were reflected by increased enzyme activity $(45.8 \pm 1.5 \mathrm{U} / \mathrm{mg}$ dry weight; $)$ measured after 45 hours of cultivation on D-glucose compared to the $\Delta$ cre1-strain $(29.1 \pm 1.8 \mathrm{U} / \mathrm{mg}$ dry weight). Interestingly, we found that in QM6a-CREI $I_{96}$ the gene expression increased with a simultaneous opening of chromatin, in particular in xyr1 and cbh1 (Figure 5a). On the other hand, we could not observe a correlation of gene expression and chromatin accessibility in the cre1 deletion strain (Figure 5b). 


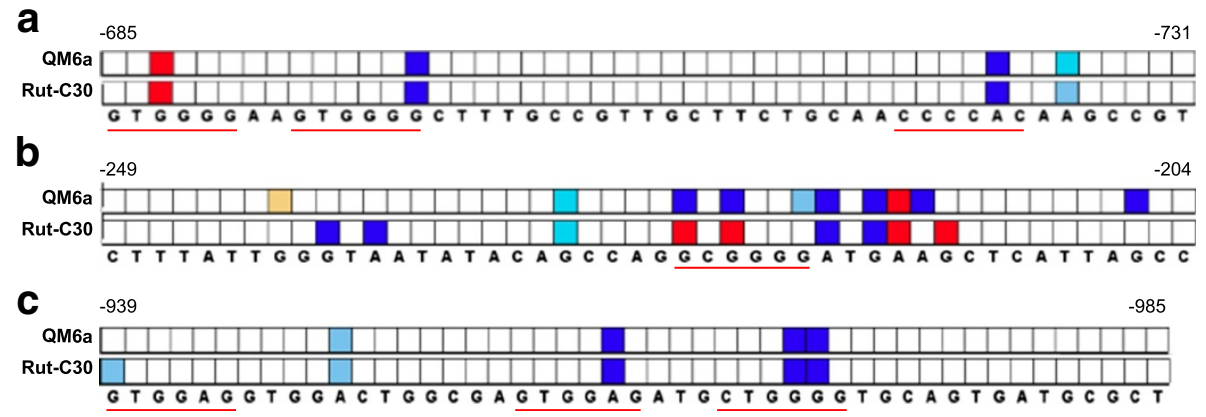

Figure 2 In vivo and in vitro footprinting analyses of URRs of CREI-target genes. The Trichoderma reesei wild-type strain QM6a and Rut-C30 were pre-grown on glycerol and then incubated on D-glucose for 3 hours followed by DMS-induced in vivo methylation. An URR bearing functional CREl sites (underlined in red) of the cbh1 (a), cbh2 (b), and xyr1 (c) genes was investigated, and methylated, naked DNA was used as the reference. Numbers indicate the position of the base upstream from ATG. Analysis of data and visualization was performed using ivFAST (in vivo footprinting analysis software tool) [27]. Only signals that are statistically different are considered. Protected bases are highlighted in red shades and hypersensitive bases are highlighted in blue shades. The three colour intensities each correspond to stronger differences between compared conditions; increasing colour intensity means more than 1.4-, 1.6-, and 1.8-fold difference in cbh1 and cbh2 (a, b), and more than 2.4-, 2.6-, and 2.8-fold difference in xyr1 (c).

To discover what this scenario looks like under inducing conditions, we applied the same experimental strategy on the two strains after pre-growth followed by incubation on sophorose for 30, 90, and 180 minutes. For $c b h 1$ and cbh2 we observed in both strains an increase in transcript levels over time, which did not correlate in either of the two strains with a simultaneous opening of chromatin (Figure 6a, b). In the xyr1 gene, transcript levels did not increase however, the chromatin became more compact over time (Figure 6a, b). Comparing the chromatin status of the two strains, the chromatin was slightly more accessible in QM6a-CREI ${ }_{96 .}$ (Figure 6a, b).

\section{CREI-96 upregulates the expression of a helicase-like transcription factor}

Due to the results obtained from the CHART-PCR analyses, we assumed that the truncated CREI-96 was involved in chromatin remodelling in both a direct and indirect
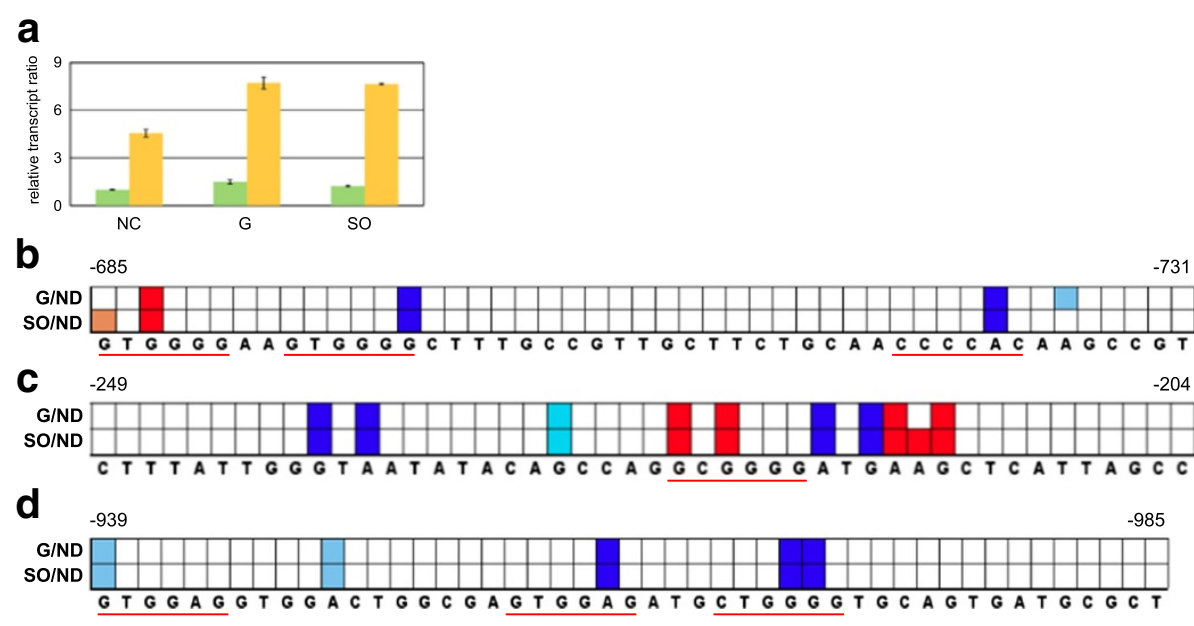

Figure 3 Analysis of the abundance of CREI-96 in Rut-C30. (a) The Trichoderma reesei wild-type strain QM6a (green bars) and Rut-C30 (yellow bars) were pre-grown on glycerol and then transferred to media supplemented with D-glucose (G), sophorose (SO) or without carbon source (NC), respectively, and incubated for 3 hours. The transcript level analysis of cre1-96 was performed by qPCR using sar1 and act genes for data normalization. Levels refer to the wild-type strain incubated without carbon source. The values are means from measurements in triplicates and three biological experiments (cultivations). Error bars indicate standard deviations. (b-d) In vivo and in vitro footprinting analysis of URRs of CREl-target genes in Rut-C30, which was pre-grown on glycerol and then incubated on D-glucose (G) or sophorose (SO) for 3 hours followed by DMS-induced in vivo methylation. An URR bearing functional CREl sites (underlined in red) of the cbh1 (b), cbh2 (c), and xyr1 (d) genes each was investigated, and methylated, naked DNA (ND) was used as the reference. Numbers indicate the position of the base upstream from ATG. Analysis of data and visualization was performed using ivFAST [27]. Colour codes are the same as in Figure 2. 


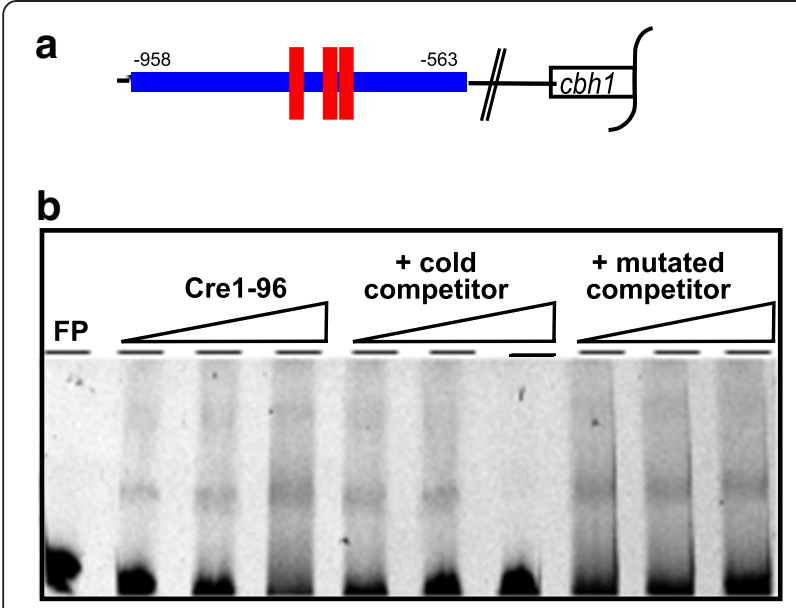

Figure 4 Analysis of the DNA-binding ability of the CREI-96 protein. (a) The EMSA was performed with a fluorescently labelled cbh1 URR fragment (4 ng) bearing three functional CREl-sites (red bars). (b) Applied were increasing amounts of the heterologously expressed and thrombin-cleaved CREI-96 protein (500, 1000, and $2000 \mathrm{nM})$, and $2000 \mathrm{nM}$ of CREl-96 together with increasing amounts (50-fold, 100 -fold, and 200-fold) of a cold competitor (unlabeled probe), and 2000 nM CREl-96 together with increasing amounts (50-fold, 100-fold, and 200-fold) of a mutated cold competitor. FP, free probe.

manner. Therefore, we searched the $T$. reesei genome for chromatin-remodelling proteins. We selected seven proteins, of which the annotation pointed to an involvement in chromatin rearrangement, for further investigation (Table 1). To determine a potential regulatory influence by CREI-96 we investigated their transcript levels in QM6a-CREI ${ }_{96}$ and the $\Delta c r e 1$-strain. Amongst the seven genes we observed one with different transcript levels, namely the gene encoding a helicase-like transcription factor (Protein ID 44747). Here we detected strong upregulation in $\mathrm{QM6a}^{-\mathrm{CREI}_{96}}$ compared to the $\Delta c r e 1$-strain, regardless of whether the samples from the D-glucose growth experiment (Figure 7a) or the sophorose replacement (Figure 7b) were investigated. We term the aforementioned gene in this study htfl (helicase-like transcription factor 1). It should be noted that in previous reports this gene was called snf2, although it is not homologous to SNF2 of Saccharomyces cerevisiae [30]. However, the increased expression of $h t f 1$ in QM6a$\mathrm{CREI}_{96}$ might be one reason for the observed change in chromatin.

\section{Discussion}

For a long time its lack of the full version of CREI has been considered to be a positive genetic property of Rut-C30 because it leads to a partial carbon catabolite de-repression. However, Rut-C30 still possesses a short version of CREI, CREI-96. During this study it became clear that this protein still has a function and influences the expression of target genes, notably, in a positive

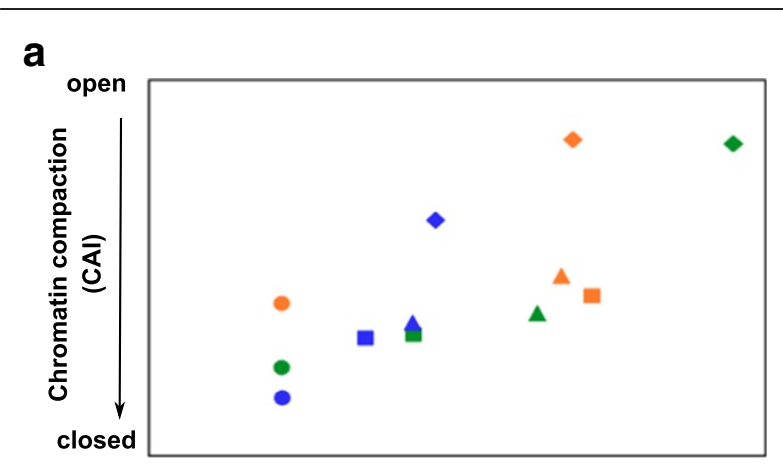

b
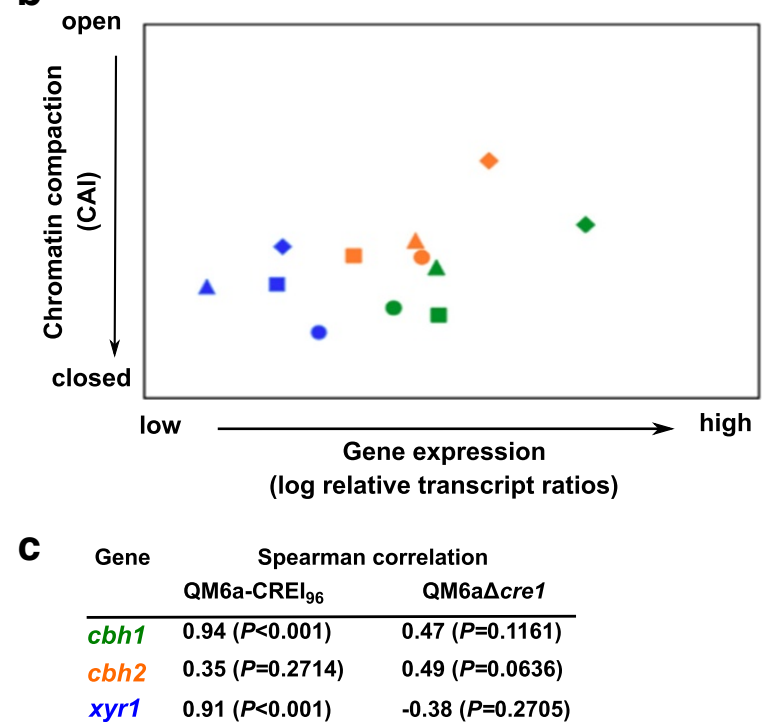

Figure 5 Transcript and CHART analysis of Trichoderma reesei CREI-mutant strains during growth on D-glucose. QM6a-CREl ${ }_{96}$ (a) and the $\Delta$ crel-strain (b) were grown on D-glucose and samples were taken after 36 (dots), 39 (boxes), 42 (triangles), 45 (diamonds) hours. The cbh1 (green), cbh2 (orange), and xyr1 (blue) genes were investigated. The gene expression analysis was performed by CDNA synthesis followed by qPCR, and transcript levels (log) are depicted on the $x$-axis. CHART-PCR was performed by Dnase I digestion followed by $\mathrm{qPCR}$, and CAls are depicted on the $y$-axis. In both cases sarl and act genes were used for data normalization and QM6a-CREl ${ }_{96}$ grown for 36 hours was the reference condition. All values are means from measurements in triplicates and three biological experiments (cultivations). Standard deviations were below 5\%. Diagrams are identically scaled. (c) Spearman's rank correlation coefficients were calculated (close to -1 indicates negative correlation; close to 0 indicates no linear correlation; close to 1 indicates positive correlation). The $P$-values for each correlation coefficient were calculated to determine significance of data (how different from zero). $P>0.05$ was considered as indicative of no linear correlation.

manner. We found three ways in which CREI-96 mechanistically exerts its role as a regulatory protein. Firstly, it acts directly on promoters of target genes. The short CREI-96 (lacking one zinc finger) can still bind to target DNA sequences in vitro, which leads in vivo to a clear protection pattern from DNA methylation at CREI- 


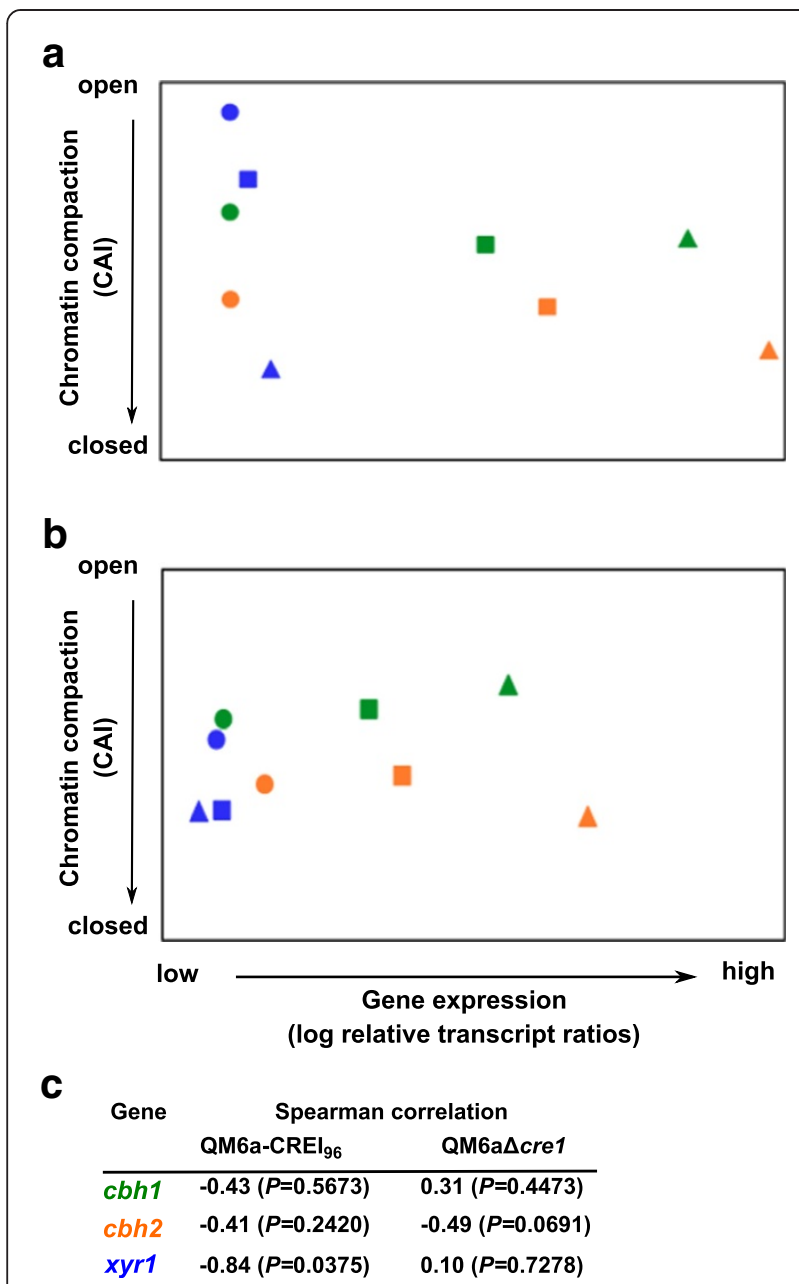

Figure 6 Transcript and CHART analysis of Trichoderma reesei CREl-mutant strains during sophorose induction. QM6a-CREl ${ }_{96}$ (a) and the $\Delta$ crel-strain (b) were pre-grown on glycerol and thereafter incubated on sophorose for 30 (dots), 90 (boxes), and 180 (triangles) minutes. The cbh1 (green), cbh2 (orange), and xyrl (blue) genes were investigated. The gene expression analysis was performed by CDNA synthesis followed by qPCR, and transcript levels are depicted on the $x$-axis. CHART-PCR was performed by Dnase I digestion followed by qPCR, and CAls are depicted on the $y$-axis. In both cases sarl and act genes were used for data normalization and QM6a-CREI $I_{96}$ incubated for 30 minutes was the reference condition. All values are means from measurements in triplicates and three biological experiments (cultivations). Standard deviations were below 5\%. Diagrams are identically scaled. (c) Spearman's rank correlation coefficients were calculated (close to -1 indicates negative correlation; close to 0 indicates no linear correlation; close to 1 indicates positive correlation). The $P$-values for each correlation coefficient were calculated to determine significance of data (how different from zero). $P>0.05$ was considered as indicative of no linear correlation.

binding sites (compare Figures 2 and 3). Obviously, contacting of CREI-binding sites in target genes by CREI-96 leads to a more open chromatin in the core promoter regions under repressing conditions (D-glucose). As expected, this effect was not observed in such a pronounced manner under inducing conditions (sophorose), as the core promoter there already has an open chromatin. If CREI-96 develops this de-regulating function by simply binding the DNA (possibly in high amounts) and thereby preventing nucleosome assembly, or if it is involved in a more complicated chromatin-DNA interaction mechanism, cannot yet be answered and this calls for further investigation.

Secondly, CREI-96 acts on the chromatin structure in an indirect way. The transcript levels of the $h t f 1$ gene encoding a chromatin-remodelling protein are upregulated in the presence of CREI-96. Notably, in the wild-type strain QM6a bearing the native CREI we found $h t f 1$ downregulated, specifically on D-glucose (data not shown). This is in good accordance with previous studies, in which this gene was reported to be repressed by CREI in the T. reesei strain QM9414 [26] and to be lowly expressed in QM6a on D-glucose [31]. It can be speculated that CREI-96 in this case reverses the antagonistic function of CREI on chromatin-remodelling proteins and even supports the opening of chromatin in an indirect way.

Thirdly, the loss of the auto-regulatory function of CREI is very likely. It should be considered that surprisingly high transcript levels of cre1-96 were detected in Rut-C30 (Figure 3a). In A. nidulans it was observed that a low steady-state level of creA mRNA can be increased within minutes by adding a repressing carbon source. While a prolonged incubation with the repressing carbon sources then led to auto-repression, the incubation on a derepressing carbon source maintained the high level [18]. In Rut-C30 the cre1-96 transcript levels on D-glucose are as high as on sophorose, indicating a loss of the mentioned native auto-repression. This might also be the reason for the generally higher abundance of transcript in Rut-C30 compared to the wild-type strain QM6a which we observed during this study. An alternative or additional explanation for the generally higher abundance of cre1-96 in Rut-C30 is mRNA stability. This may arise from less stability of the mRNA coding for the full-length protein, or a general increase in mRNA stability speculated to be caused by two mutations (protein ID 110423 and 66895) identified in the direct ancestor of Rut-C30 (NG14) [11]. However, it can be assumed that in Rut-C30 this results in high amounts of CREI-96 present in the cell, which is still able to bind DNA, thereby possibly changing the chromatin status in CREI-target genes.

Even if in previous studies truncated forms of CREI have been investigated, interestingly, none of them reported a positive effect of the shorter CREI version compared to a deletion of CREI. This is most likely due to a different experimental design applied. Nakari-Setälä et al. reported similar cellulase activities (volumetrically given) of strains bearing a deletion or a truncation of CREI when grown on lactose [32]. However, the investigated strains exhibited different growth behaviors (biomass formation and $\mathrm{CO}_{2}$ - 
Table 1 Investigated candidate genes encoding ATP-dependent chromatin remodelling factors

\begin{tabular}{lll}
\hline Protein ID & Annotation $^{\mathbf{a}^{2}}$ & Provisory name \\
\hline 44747 & Helicase-like transcription factor HLTF/DNA helicase RAD5, DEAD-box superfamily $^{2}$ & htf1 (snf2) \\
57935 & Chromatin remodelling complex SWI/SNF, component SWI2 and related ATPases & snf2-like \\
& (DNA/RNA helicase superfamily)/ATPase & rsC8 \\
57557 & Chromatin remodelling factor subunit and related transcription factors & isw1 \\
109526 & Chromatin remodelling complex WSTF-ISWl, small subunit & isw2 \\
50539 & Chromatin remodelling complex WSTF-ISWI, small subunit & ino80 \\
58928 & SNF2 family DNA-dependent ATPase & cdh1 \\
\hline
\end{tabular}

according to TRIRE Joint Genome Institute - JGI - Trichoderma reesei v2.0 database.

levels) [32]. In order to exclude any growth effects we studied a resting cell system under inducing conditions (sophorose). Such a highly standardized transfer experiment allows determination of the mechanistic influence of an isolated phenomenon (presence of CREI-96 or lack of CREI).

We also investigated growth conditions on D-glucose as this is certainly industrially relevant (enzyme production on high glucose-containing inducers). In this case we also observed differences in transcript levels between the two CREI mutant strains, which is in accordance with the

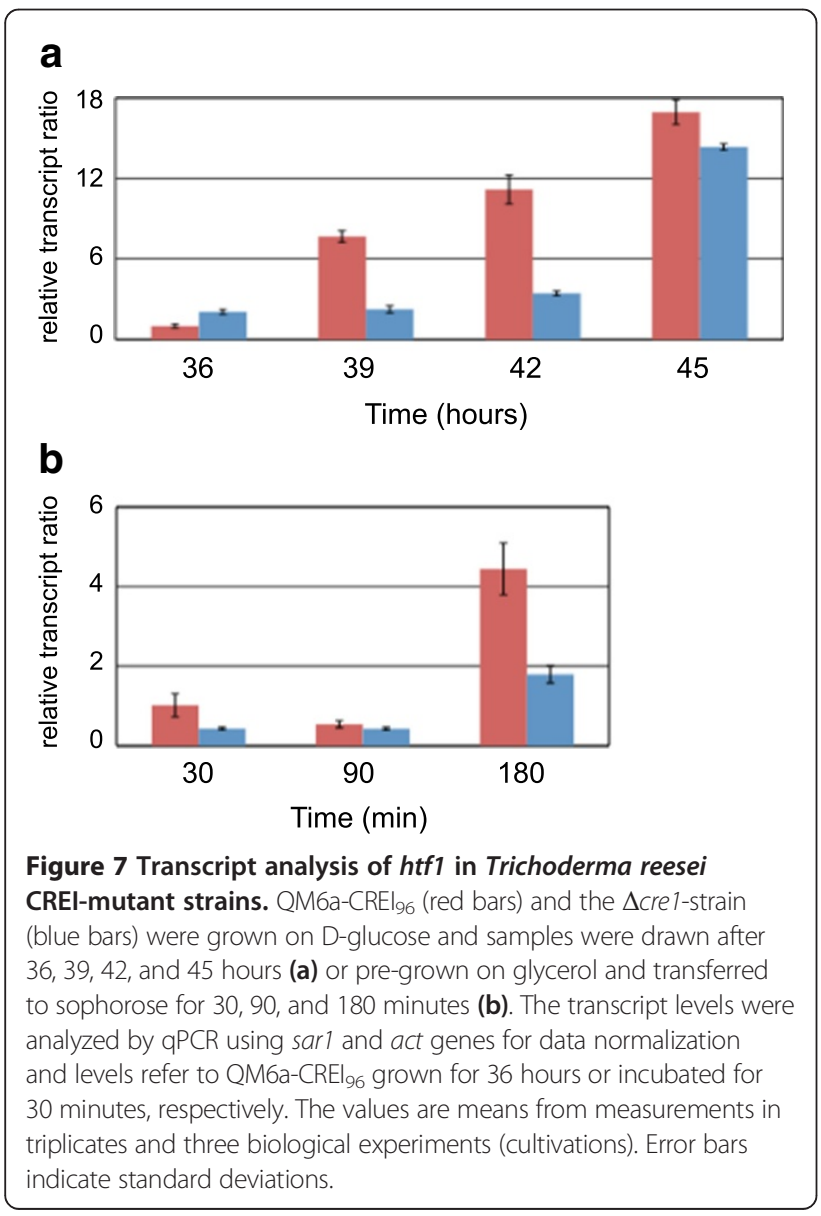

results of a Northern analysis of $c b h 1$ on glucose by Nakari-Setälä et al. [32] and of the cbh1 transcript level analysis by Ries et al. [24].

We noticed that the increased transcript levels in strain QM6a-CREI ${ }_{96}$ corresponded with an opening of chromatin on D-glucose, which was not found on sophorose. We propose that this different observation is likely due to the fact that the remodelling of chromatin usually becomes necessary under repressing conditions, while under inducing conditions certain regulatory factors and/ or mechanisms have already ensured the open chromatin status. Obviously, the differences between the two CREI mutant strains observed on sophorose are primarily related to induction, chromatin opening, and expression of XYRI. During early-stage induction (30 minutes) the presence of sophorose leads to a rapid and higher chromatin accessibility of $x y r 1$ in QM6a-CREI ${ }_{96}$, which subsequently results in higher xyr1 transcript levels (Figure 6). It was previously reported that the level of xyr1 expression is directly linked to the expression of $c b h 1$ and cbh2 [6]. Accordingly, we detected higher cbh1 and $c b h 2$ transcript levels in the QM6a-CREI 96 on sophorose.

\section{Conclusions}

From the result obtained during this study we conclude that the truncated version of CREI present in Rut-C30 (CREI-96) should be considered as a discrete transcription factor with different properties than CREI. It acts in a direct manner on target URRs, but also contributes indirectly to a more open chromatin status by regulating a chromatin remodeler. Altogether it should be considered that Rut-C30 not only lacks CREI and the thereby mediated CCR, but also gains the now positively acting regulatory protein CREI-96.

Orthologs of CREI regulate CCR in numerous filamentous fungi including those used in biofuel production. Unfortunately, a simple deletion of cre1 does not only lead to the desired release from CCR, but also to severe growth impairment. Consequently, molecular strain design can alternatively be based on the described CREI-96 truncation. This strategy does on one hand avoid growth 
deficiencies and the accompanying loss of productivity, and on the other hand additionally leads to a chromatin remodelling effect that results in increased expression of PCW-degrading enzymes.

\section{Materials and Methods Fungal strains}

The following T. reesei strains were used throughout this study: the wild-type strain QM6a (ATCC 13631), Rut-C30, which was described as a high yielding cellulase mutant of QM6a (ATCC 56765) [10], a cre1 deletion in QM6a $(\Delta c r e 1)$ [32], a $83 \mathrm{~kb}$ deletion corresponding to the large deletion in scaffold 15 of Rut-C30 in QM6a $\Delta$ tmus53 ( $\Delta$ scaff15) $[13,33]$, as well as a QM6a $\Delta$ tmus53 strain bearing the truncated cre1 of Rut-C30 (QM6a-CREI $I_{96}$ ) constructed during this study. All strains were maintained on malt extract agar or potato-dextrose-agar.

\section{Growth conditions}

For carbon source replacement experiments mycelia were pre-cultured in $1 \mathrm{~L}$ Erlenmeyer flasks on a rotary shaker $(180 \mathrm{rpm})$ at $30^{\circ} \mathrm{C}$ for 24 hours in $250 \mathrm{~mL}$ of Mandels-Andreotti (MA) medium [34] supplemented with $1 \%(\mathrm{w} / \mathrm{v})$ glycerol as sole carbon source. A total of $10^{9}$ conidia per litre (final concentration) were used as inoculum. Pre-grown mycelia were washed and equal amounts were resuspended in $20 \mathrm{ml}$ MA media containing $1 \%(\mathrm{w} / \mathrm{v})$ D-glucose or $2 \mathrm{mM}$ sophorose (Serva Electrophoresis, Heidelberg, Germany) as sole carbon source, or no carbon source respectively, and incubated for 30 minutes to 3 hours. For direct cultivation experiments the conidia were incubated in $250 \mathrm{~mL}$ Erlenmeyer flasks on a rotary shaker $(180 \mathrm{rpm})$ at $30^{\circ} \mathrm{C}$ for 45 hours in $50 \mathrm{~mL}$ of MA medium supplemented with $1 \%(\mathrm{w} / \mathrm{v}) \mathrm{D}$-glucose as sole carbon source. Samples were derived from three biological replicates and were pooled before RNA extraction and chromatin digestion.

\section{Replacement of CREI in T. reesei QM6a}

Transformation of $T$. reesei QM6a $\Delta \mathrm{tmus} 53$ [33] was performed using two overlapping $3 \mathrm{~kb}-\mathrm{DNA}$ fragments. The first fragment consisted of the truncated cre1 of Rut-C30 amplified with primers RG186 and RG187 using genomic DNA as template and 3 '-half of the expression cassette bearing the Escherichia coli hph marker gene amplified with primers RG188 and hph3' fw using the plasmid $\mathrm{pRLMex}_{30}$ [35] as template. The second fragment consisted of the $5^{\prime}$-half of the expression cassette bearing the E. coli hph marker gene amplified with primers hph $5^{\prime}$ rev and RG189 using pRLMex 30 as template and the cre1 3'flank from Rut-C30 amplified with primers RG190 and RG191 using genomic DNA as template. Protoplast transformation of QM6a was performed as described in United States patent number 8,323,931 using $2.5 \mu \mathrm{g}$ of each DNA fragment in a co-transformation. Genomic integration of the full construct (cre1-96::hph) into the cre1 locus was confirmed by southern blot analysis and DNA sequencing (Microsynth, Balgach, Switzerland).

\section{In vivo footprinting}

In vivo methylation using DMS followed by ligationmediated PCR was performed as described previously [27]. FAM (fluorescein amidite)-labelled fragments were analyzed by capillary gel electrophoresis (Microsynth) and results were analyzed using ivFAST [27].

\section{Analysis of transcript levels}

Fungal mycelia were homogenized in $1 \mathrm{~mL}$ of peqGOLDTriFast DNA/RNA/protein purification system reagent (PEQLAB Biotechnologie, Erlangen, Germany) using a FastPrep(R)-24 cell disrupter (MP Biomedicals, Santa Ana, California, United States). RNA was isolated according to the manufacturer's instructions, and the concentration was measured using the NanoDrop 1000 (Thermo Scientific, Waltham, Massachusetts, United States). Synthesis of cDNA from mRNA was carried out using the RevertAidTM H Minus First Strand cDNA Synthesis Kit (Thermo Scientific, Waltham, Massachusetts, United States) according to the manufacturer's instructions. Quantitative PCRs were performed in a Rotor-Gene Q system (Qiagen, Hilden, Germany). All reactions were performed in triplicate. The amplification mixture (final volume $15 \mu \mathrm{L}$ ) contained 7.5 $\mu \mathrm{L} 2 \times$ iQ SYBR Green Mix (Bio-Rad, Hercules, USA), $100 \mathrm{nM}$ forward and reverse primer, and $2.5 \mu \mathrm{L}$ cDNA (diluted 1:20). Primer sequences are provided in Table 2. Cycling conditions and control reactions were performed as described previously [36]. Data normalization using sar 1 and act as reference genes and calculations were performed as published previously [36].

\section{Plasmid construction}

A 307 bp fragment was amplified from Rut-C30 genomic DNA using primers RG182 and RG178 and was inserted into the expression vector pGEX-4 T-2 (GE Healthcare Life Sciences, Little Chalfont, Buckinghamshire, United Kingdom) via $\mathrm{BamHI} / \mathrm{XhoI}$ digestion yielding the plasmid pGEX-cre1-RG182 for heterologous expression of GST (glutathione S-transferase)-fused CREI-96.

\section{Protein expression and purification}

E. coli BL21(DE3)pLysS (Promega, Madison, Wisconsin, United States) carrying pGEX-cre1-RG182 was cultivated in a $1 \mathrm{~L}$ Erlenmeyer flask on a rotary shaker $(200 \mathrm{rpm})$ at $37^{\circ} \mathrm{C}$ in $300 \mathrm{~mL}$ LB medium supplemented with ampicillin $(50 \mu \mathrm{g} / \mathrm{mL})$ until an $\mathrm{OD}_{600}$ of 0.5 was reached. Protein expression was induced by adding IPTG to a final concentration of $0.5 \mathrm{mM}$ followed by an incubation at $37^{\circ} \mathrm{C}$ for 3 hours. The cells were harvested by centrifugation and 
Table 2 Oligonucleotides used in this study

\begin{tabular}{|c|c|c|}
\hline Name & Sequence $\left(5^{\prime}-3^{\prime}\right)$ & Usage \\
\hline RG53 & GAATTCAGATC & iv-FP, oligo-short \\
\hline RG54 & GCGGTGACCCGGGAGATCTGAATTC & iv-FP, oligo-long \\
\hline RG89 & [6-FAM]GTAGAGGCATGTTGTGAATCTGTGTCGGG & iv-FP, cbh1oligo3fw, EMSA \\
\hline RG90 & [6-FAM]GGTTGTATGCAAAACGCTCCGAGTCAGAC & iv-FP, cbh1oligo3rev, EMSA \\
\hline RG215 & CCAACGGCTTGTGGGGTTGCAGAAGCAACGGCAAAGCCCCACTTCCCCACGTTTGTTTCT & EMSA \\
\hline RG216 & AGAAACAAACGTGGGGAAGTGGGGCTTTGCCGTTGCTTCTGCAACCCCACAAGCCGTTGG & \\
\hline RG221 & CCAACGGCTTGTTGGGTTGCAGAAGCAACGGCAAAGCCCAACTTCCCAACGTTTGTTCT ${ }^{\mathbf{b}}$ & \\
\hline RG222 & AGAAACAAACGTTGGGAAGTTGGGCTTTGCCGTTGCTTCTGCAACCCAACAAGCCGTTGG & \\
\hline RG178 & TATCTCGAGTTTAGAAAAAAAAGCAGGT & pGEX-cre1-RG182 construction \\
\hline RG182 & ATTGGATCCATGCAACGAGCACAGTCTGCCGT & \\
\hline RG186 & TTGAGTGCAGACGTGTGTGTAATCTT & Construction of QM6a-CREI $I_{96}$ \\
\hline RG187 & CCCTCCTITGTTAGAAAAAAAAGCAGGTAATGG & \\
\hline RG188 & TाTाTCTAACAAAGGAGGGAGACGAGGTTG & \\
\hline RG189 & CCTACATTGGATAACGGTGAGACTAGCGGCC & \\
\hline RG190 & TCACCGTTATCCAATGTAGGTAAGTAGTAAGGG & \\
\hline RG191 & GAATCAGTATTTTCTCATCTCCTTG & \\
\hline hph3'_fw & GACCTGCCTGAAACCGAACTG & \\
\hline hph5'_rev & GAAGAAGATGTTGGCGACCTCG & \\
\hline actfw & TGAGAGCGGTGGTATCCACG & qPCR \\
\hline actrev & GGTACCACCAGACATGACAATGTTG & \\
\hline sar1fw & TGGATCGTCAACTGGTTCTACGA & \\
\hline sar1rev & GCATGTGTAGCAACGTGGTCTTT & \\
\hline cbh1f & GATGATGACTACGCCAACATGCTG & \\
\hline cbh1r & ACGGCACCGGGTGTGG & \\
\hline $\mathrm{cbh} 2 \mathrm{f}$ & CTATGCCGGACAGTTTGTGGTG & \\
\hline $\mathrm{cbh} 2 \mathrm{r}$ & GTCAGGCTCAATAACCAGGAGG & \\
\hline xyr1f & CCCATTCGGCGGAGGATCAG & \\
\hline xyr1r & CGAATTCTATACAATGGGCACATGGG & \\
\hline $44747 f$ & GCTCGAGCTGCAAGACAAGA & \\
\hline $44747 r$ & GCGGAGATCCATGAGCTTCT & \\
\hline epiactinTr_f & СТТСССТССТТССТСССССТССАС & act CHART, region -226 to +24 \\
\hline epiactinTr_r & GCGACAGGTGCACGTACCCTCCATT & \\
\hline episar1Tr_f & GTCAGGAAATGCCGCACAAGCAAGA & sar1 CHART, region -490 to -224 \\
\hline episar1Tr_r & TGTGTTTACCGCCTTGGCCTTTGG & \\
\hline epicbh1_2Tr_f & GGATCGAACACACTGCTGCCTITAC & cbh1 CHART, region -301 to -27 \\
\hline epicbh1_2Tr_r & GGTTTCTGTGCCTCAAAAGATGGTG & \\
\hline epicbh2_2Tr_f & TGCAGCGCAACACTACACGCAACAT & cbh2 CHART, region -355 to -62 \\
\hline epicbh2_2Tr_r & TGCGCCTCATACAGGGTCACAGTCC & \\
\hline epixyr1_2Tr_f & CCGACAGCAGCAGTAGTCAGGTTT & xyr1 CHART, region -216 to +35 \\
\hline epixyr1_2Tr_r & TAGGCAGAATAGCGACGGAGAGGAT & \\
\hline
\end{tabular}

altalic letters indicate a CREI-binding site (5'-SYGGRG-3').

${ }^{\mathbf{b}}$ Bold letters indicate the introduced mutation in the CREI-binding site (5'-SYTGRG-3'). 
stored at $-20^{\circ} \mathrm{C}$ overnight. GST-fusion protein (theoretical molecular weight $37 \mathrm{kDa}$ ) was purified from $E$. coli cell lysates using Glutathione-Superflow Resin (Qiagen, Hilden, Germany) according to the manufacturer's instructions.

\section{Electrophoretic mobility shift assay (EMSA)}

A 400-bp PCR product comprising the part of the $5^{\prime}$ URR region of $c b h 1$, which contains 3 CREI-binding sites, was obtained with FAM-labelled primers RG89 and RG90 and used as probe (Table 2). The protein-DNA binding assay and non-denaturing polyacrylamide gel electrophoresis were performed essentially as previously described [37]. Binding was achieved by incubating increasing amounts of heterologously expressed, thrombin-cleaved CREI-96 (500 nM, $1000 \mathrm{nM}$, and $2000 \mathrm{nM}$ ) with $4 \mathrm{ng}$ of the labelled, double-stranded DNA fragment in GST elution buffer $\left(10\right.$ minutes at $\left.22^{\circ} \mathrm{C}\right)$. Fluorescence and image analysis of the gels was carried out using a Typhoon 8600 variable mode imager (Amersham Bioscience, part of GE Healthcare, Little Chalfont, Buckinghamshire, United Kingdom). Competition experiments were performed using $2000 \mathrm{nM}$ of protein together with increasing amounts (50-fold, 100-fold, and 200-fold) of either a cold competitor (an unlabeled double-stranded DNA probe obtained by annealing primers RG215 and RG216) or a mutated cold competitor (an unlabeled doublestranded DNA probe obtained by annealing primers RG221 and RG222). This probe bears in all three CREI-binding sites a mutation from 5 ' - SYGGRG-3' to 5'-SYTGRG-3', which was previously shown to prevent the binding of CREI in vivo and in vitro [21].

\section{Determination of cellulase activity}

Cellulase activity in the culture supernatants was determined using AZCL HE-Cellulose (Megazyme International, Bray, Ireland) in $25 \mathrm{mM}$ sodium acetate buffer $\mathrm{pH} 4.5$ according to the manufacturer's instructions. To measure biomass (dry weight), the cultures were harvested by filtration, washed with an equal volume of $0.8 \% \mathrm{NaCl}$ solution, dried at $80^{\circ} \mathrm{C}$ for 24 hours, and weighed. Samples from two biological replicates and two technical replicates were measured.

\section{Chromatin accessibility real-time PCR (CHART-PCR)}

DNase I digestion of chromatin and DNA extraction were carried out as described by Gonzalez and Scazzocchio [38] with minor modifications. Mycelia were harvested by filtration, pressed dry with filter paper, frozen in liquid nitrogen, and ground to a fine powder. Portions (100 mg) of the powder were suspended in $1 \mathrm{~mL}$ aliquots of nuclease digestion buffer $(250 \mathrm{mM}$ sucrose, $60 \mathrm{mM} \mathrm{KCl}, 15 \mathrm{mM}$ $\mathrm{NaCl}, 0.05 \mathrm{mM} \mathrm{CaCl}, 3 \mathrm{mM} \mathrm{MgCl}_{2}, 0.5 \mathrm{mM}$ DTT, and $15 \mathrm{mM}$ Tris- $\mathrm{HCl}$ at $\mathrm{pH} 7.5$ ), and $100-\mu \mathrm{L}$ samples of the digestion mixture were incubated with $10 \mathrm{U}$ of
RQ1 RNase-free DNase I (Promega, Madison, Wisconsin, United States) for 2.5 minutes at $37^{\circ} \mathrm{C}$. The reaction was stopped by adding $100 \mu \mathrm{L}$ of $40 \mathrm{mM}$ EDTA and $2 \%$ SDS, followed by two rounds of phenol-chloroform extraction and one round of chloroform extraction. Samples were then treated with $10 \mu \mathrm{g} / \mathrm{mL}$ of RNase A for 15 minutes at $37^{\circ} \mathrm{C}$ and precipitated with ethanol. DNA pellets were suspended in $100 \mu \mathrm{L}$ of $5 \mathrm{mM}$ Tris- $\mathrm{HCl}$ at $\mathrm{pH}$ 7.5. A control without DNase I was included to monitor endonuclease activity. qPCR analysis of the DNase I-treated samples was performed to measure the relative abundance of target regions. PCRs were performed in a Rotor-Gene Q system (Qiagen, Hilden, Germany). All reactions were performed in triplicate. The amplification mixture (final volume $20 \mu \mathrm{L}$ ) contained $10 \mu \mathrm{L} 2 \times$ iQ SYBR Green Mix (Bio-Rad, Hercules, USA), $200 \mathrm{nM}$ forward and reverse primers and $10 \mathrm{ng}$ of DNA. Primer sequences are provided in Table 2. Cycling conditions were as follows: 3 minutes initial denaturation at $95^{\circ} \mathrm{C}$, followed by 40 cycles of 15 seconds at $95^{\circ} \mathrm{C}$ and 60 seconds at $60^{\circ} \mathrm{C}$. The amount of intact input DNA of each sample was calculated by comparing the threshold values of the PCR amplification plots with a standard curve generated for each primer set using serial dilutions of genomic, undigested DNA. The chromatin accessibility index (CAI) was defined as:

$$
\mathrm{CAI}=1 /(\mathrm{Ds} /((\mathrm{Dc} 1+\mathrm{Dc} 2) / 2))
$$

where Ds is the amount of intact DNA detected for each target region and Dc1 and Dc2 are the amounts of intact DNA detect for the promoter regions of sar1 and act respectively, used as reference genes for normalization.

To access the relationship between the CAI and the transcript level, a two-tailed Spearman's rank correlation coefficient was determined for each gene analyzed. The $P$-value for each correlation coefficient was calculated to determine the significance of the data.

\section{Selection of investigated genes encoding chromatin remodelling factors}

Genes were selected by direct searching for SNF2, ISW1, ISW2, INO80, CDH1, RSC8 (the most prominent chromatin remodelling-related proteins characterized in yeast) in the $T$. reesei genome database [39]. Additional candidate genes were obtained by using BLASTp (basic local alignment search tool) search in the NCBI database employing respective $S$. cerevisiae sequences as baits to identify similar sequences in filamentous fungi. Subsequently, these sequences were used in a BLAST search in the $T$. reesei database.

\section{Abbreviations}

BLAST: Basic local alignment search tool; CAl: Chromatin accessibility index: CHART-PCR: Chromatin accessibility real-time PCR; CREl: Carbon catabolite repressor 1; DMS: Dimethyl sulphate; EMSA: Electrophoretic mobility shift assay; GST: Glutathione S-transferase; ivFAST: in vivo footprinting analysis 
software tool; MA: Mandels-Andreotti; PCW: Plant cell wall; RT-qPCR: Reverse transcription quantitative $P C R$; URR: Upstream regulatory region; XYRI: Xylanase regulator 1 .

\section{Competing interests}

The authors declare that they have no competing interests.

\section{Authors' contributions}

RG constructed the strain bearing the truncated version of CREI. TMMS carried out transcript analyses, CHART-PCR, and EMSA. AR carried out the in vivo footprinting analyses. MJPF participated in conception of the study and revised the manuscript critically. RLM participated in conception of the study. ARMA participated in conception of the study, supervised the experiments, and prepared the manuscript. All authors read and approved the final manuscript.

\section{Acknowledgements}

This study was supported by two grants from the Austrian Science Fund (FWF; grant numbers V232-B20 and P24851) given to ARM-A. The recombinant QM6a strains $\Delta c r e 1$ and $\Delta$ scaff15 used in this study were originally created at VTT Technical Research Centre of Finland and their receipt is gratefully acknowledged. We thank René Mayer for his help in performing appropriate statistical tests.

\section{Author details}

'Department for Biotechnology and Microbiology, Institute of Chemical Engineering, Vienna University of Technology, Gumpendorfer Str. 1a, A-1060 Wien, Austria. ${ }^{2}$ Department of Genetics and Morphology, Institute of Biological Sciences, University of Brasília, Campus Universitário Darcy Ribeiro, 70910-900 Brasília, DF, Brazil.

Received: 5 June 2014 Accepted: 22 August 2014

Published online: 11 September 2014

\section{References}

1. Martinez D, Berka RM, Henrissat B, Saloheimo M, Arvas M, Baker SE, Chapman J, Chertkov O, Coutinho PM, Cullen D, Danchin EG, Grigoriev IV, Harris P, Jackson M, Kubicek CP, Han CS, Ho I, Larrondo LF, de Leon AL, Magnuson JK, Merino S, Misra M, Nelson B, Putnam N, Robbertse B, Salamov AA, Schmoll M, Terry A, Thayer N, Westerholm-Parvinen A, et al: Genome sequencing and analysis of the biomass-degrading fungus Trichoderma reesei (syn. Hypocrea jecorina). Nat Biotechnol 2008, 26:553-560.

2. Teeri T, Salovouri I, Knowles J: The molecular cloning of the major cellulase gene from Trichoderma reesei. Biotechnology 1983, 1:696-699.

3. Buchert J, Oksanen T, Pere J, Siika-aho M, Suurnäkki A, Viikari L: Applications of Trichoderma reesei Enzymes in the Pulp and Paper Industry. In Trichoderma \& Gliocladium, Volume 2. Edited by Kubicek CP, Harman GE. London: Taylor \& Francis; 1998:343-357.

4. Galante YM, de Conti A, Monteverdi R: Application of Trichoderma Enzymes in the Food and Feed Industries. In Trichoderma \& Gliocladium, Volume 2. Edited by Kubicek CP, Harman GE. London: Taylor \& Francis; 1998:327-342.

5. Galante YM, de Conti A, Monteverdi R: Application of Trichoderma Enzymes in the Textile Industry. In Trichoderma \& Gliocladium, Volume 2. Edited by Kubicek CP, Harman GE. London: Taylor \& Francis; 1998:311-325.

6. Derntl C, Gudynaite-Savitch L, Calixte S, White T, Mach RL, Mach-Aigner AR: Mutation of the Xylanase regulator 1 causes a glucose blind hydrolase expressing phenotype in industrially used Trichoderma strains. Biotechnol Biofuels 2013, 6:62

7. Peterson R, Nevalainen H: Trichoderma reesei RUT-C30 - thirty years of strain improvement. Microbiology 2012, 158:58-68

8. Montenecourt BS, Eveleigh DE: Semiquantitative plate assay for determination of cellulase production by Trichoderma viride. Appl Environ Microbiol 1977, 33:178-183.

9. Montenecourt BS, Eveleigh DE: Preparation of mutants of Trichoderma reesei with enhanced cellulase production. Appl Environ Microbiol 1977 34:777-782.

10. Montenecourt BS, Eveleigh DE: Selective Screening Methods for the Isolation of High Yielding Cellulase Mutants of Trichoderma reesei. In Hydrolysis of Cellulose: Mechanisms of Enzymatic and Acid Catalysis, Volume 181. Edited by Brown RD, Jurasek L. Washington DC: American Chemical Society; 1979:289-301.
11. Le Crom S, Schackwitz W, Pennacchio L, Magnuson JK, Culley DE, Collett JR, Martin J, Druzhinina IS, Mathis H, Monot F, Seiboth B, Cherry B, Rey M, Berka R, Kubicek CP, Baker SE, Margeot A: Tracking the roots of cellulase hyperproduction by the fungus Trichoderma reesei using massively parallel DNA sequencing. Proc Natl Acad Sci U S A 2009, 106:16151-16156.

12. Seidl V, Gamauf C, Druzhinina IS, Seiboth B, Hartl L, Kubicek CP: The Hypocrea jecorina (Trichoderma reesei) hypercellulolytic mutant RUT C30 lacks a $85 \mathrm{~kb}$ (29 gene-encoding) region of the wild-type genome. BMC Genomics 2008, 9:327.

13. Vitikainen M, Arvas M, Pakula T, Oja M, Penttilä M, Saloheimo M: Array comparative genomic hybridization analysis of Trichoderma reesei strains with enhanced cellulase production properties. BMC Genomics 2010, 11:441.

14. Strauss J, Mach RL, Zeilinger S, Hartler G, Stoffler G, Wolschek M, Kubicek CP: $\mathrm{Cre1}$, the carbon catabolite repressor protein from Trichoderma reesei. FEBS Lett 1995, 376:103-107.

15. IImén $M$, Thrane C, Penttilä M: The glucose repressor gene cre1 of Trichoderma: isolation and expression of a full-length and a truncated mutant form. Mol Gen Genet 1996, 251:451-460.

16. Bailey C, Arst HN Jr: Carbon catabolite repression in Aspergillus nidulans. Eur J Biochem 1975, 51:573-577.

17. Drysdale MR, Kolze SE, Kelly JM: The Aspergillus niger carbon catabolite repressor encoding gene, creA. Gene 1993, 130:241-245.

18. Strauss J, Horvath HK, Abdallah BM, Kindermann J, Mach RL, Kubicek CP: The function of CreA, the carbon catabolite repressor of Aspergillus nidulans, is regulated at the transcriptional and post-transcriptional level. Mol Microbiol 1999, 32:169-178.

19. Nehlin JO, Carlberg M, Ronne H: Control of yeast GAL genes by MIG1 repressor: a transcriptional cascade in the glucose response. $E M B O$ 1991, 10:3373-3377.

20. IImén M, Onnela ML, Klemsdal S, Keranen S, Penttilä M: Functional analysis of the cellobiohydrolase I promoter of the filamentous fungus Trichoderma reesei. Mol Gen Genet 1998, 257:386.

21. Mach RL, Strauss J, Zeilinger S, Schindler M, Kubicek CP: Carbon catabolite repression of xylanase I (xyn1) gene expression in Trichoderma reesei. Mol Microbiol 1996, 21:1273-1281

22. Mach-Aigner AR, Pucher ME, Steiger MG, Bauer GE, Preis SJ, Mach RL: Transcriptional regulation of $x y r 1$, encoding the main regulator of the xylanolytic and cellulolytic enzyme system in Hypocrea jecorina. Appl Environ Microbiol 2008, 74:6554-6562.

23. Stricker AR, Grosstessner-Hain K, Würleitner E, Mach RL: Xyr1 (xylanase regulator 1) regulates both the hydrolytic enzyme system and D-xylose metabolism in Hypocrea jecorina. Eukaryot Cell 2006, 5:2128-2137.

24. Ries L, Belshaw NJ, IImén M, Penttilä ME, Alapuranen M, Archer DB: The role of CRE1 in nucleosome positioning within the cbh1 promoter and coding regions of Trichoderma reesei. Appl Microbiol Biotechnol 2014, 98:749-762.

25. Zeilinger S, Schmoll M, Pail M, Mach RL, Kubicek CP: Nucleosome transactions on the Hypocrea jecorina (Trichoderma reesei) cellulase promoter cbh2 associated with cellulase induction. Mol Genet Genomics 2003, 270:46-55.

26. Portnoy $T$, Margeot A, Linke R, Atanasova L, Fekete E, Sandor E, Hartl L, Karaffa L, Druzhinina IS, Seiboth B, Le Crom S, Kubicek CP: The CRE1 carbon catabolite repressor of the fungus Trichoderma reesei: a master regulator of carbon assimilation. BMC Genomics 2011, 12:269.

27. Gorsche R, Jovanovic B, Gudynaite-Savitch L, Mach RL, Mach-Aigner AR: A highly sensitive in vivo footprinting technique for condition-dependent identification of cis elements. Nucleic Acids Res 2014, 42:e1.

28. Cziferszky A, Mach RL, Kubicek CP: Phosphorylation positively regulates DNA binding of the carbon catabolite repressor Cre1 of Hypocrea jecorina (Trichoderma reesei). J Biol Chem 2002, 277:14688-14694.

29. Manfield IW, Reynolds LA, Gittins J, Kneale GG: The DNA-binding domain of the gene regulatory protein AreA extends beyond the minimal zinc-finger region conserved between GATA proteins. Biochim Biophys Acta 2000, 1493:325-332.

30. Abrams E, Neigeborn L, Carlson M: Molecular analysis of SNF2 and SNF5, genes required for expression of glucose-repressible genes in Saccharomyces cerevisiae. Mol Cell Biol 1986, 6:3643-3651.

31. Ries L, Pullan ST, Delmas S, Malla S, Blythe MJ, Archer DB: Genome-wide transcriptional response of Trichoderma reesei to lignocellulose using RNA sequencing and comparison with Aspergillus niger. BMC Genomics 2013, 14:541.

32. Nakari-Setälä T, Paloheimo M, Kallio J, Vehmaanperä J, Penttilä M, Saloheimo M: Genetic modification of carbon catabolite repression in Trichoderma 
reesei for improved protein production. Appl Environ Microbiol 2009, 75:4853-4860

33. Steiger MG, Vitikainen M, Uskonen P, Brunner K, Adam G, Pakula T, Penttilä M, Saloheimo M, Mach RL, Mach-Aigner AR: Transformation system for Hypocrea jecorina (Trichoderma reesei) that favors homologous integration and employs reusable bidirectionally selectable markers. Appl Environ Microbiol 2011, 77:114-121.

34. Mandels M: Applications of cellulases. Biochem Soc Trans 1985, 13:414-416.

35. Mach RL, Schindler M, Kubicek CP: Transformation of Trichoderma reesei based on hygromycin B resistance using homologous expression signals. Curr Genet 1994, 25:567-570.

36. Steiger MG, Mach RL, Mach-Aigner AR: An accurate normalization strategy for RT-qPCR in Hypocrea jecorina (Trichoderma reesei). J Biotechnol 2010, 145:30-37.

37. Stangl H, Gruber F, Kubicek CP: Characterization of the Trichoderma reesei cbh2 promoter. Curr Genet 1993, 23:115-122.

38. Gonzalez R, Scazzocchio C: A rapid method for chromatin structure analysis in the filamentous fungus Aspergillus nidulans. Nucleic Acids Res 1997, 25:3955-3956.

39. Joint Genome Institute: Trichoderma reesei v2.0. http://genome.jgi-psf.org/ Trire2/Trire2.home.html.

doi:10.1186/s13068-014-0129-3

Cite this article as: Mello-de-Sousa et al: A truncated form of the Carbon catabolite repressor 1 increases cellulase production in Trichoderma reesei. Biotechnology for Biofuels 2014 7:129.

\section{Submit your next manuscript to BioMed Central and take full advantage of:}

- Convenient online submission

- Thorough peer review

- No space constraints or color figure charges

- Immediate publication on acceptance

- Inclusion in PubMed, CAS, Scopus and Google Scholar

- Research which is freely available for redistribution 